University of Warwick institutional repository: http://go.warwick.ac.uk/wrap This paper is made available online in accordance with publisher policies. Please scroll down to view the document itself. Please refer to the repository record for this item and our policy information available from the repository home page for further information.

To see the final version of this paper please visit the publisher's website. Access to the published version may require a subscription.

Author(s): M. TSALAS, S. C. CHAPMAN and G. ROWLANDS Article Title: The stability of charged-particle motion in sheared magnetic reversals

Year of publication: 2001

Link to published version:

http://dx.doi.org/10.1017/S0022377801001039

Publisher statement: None 


\title{
The stability of charged-particle motion in sheared magnetic reversals
}

\author{
M. TSALAS, S. C. CHA PMAN and G. ROWLANDS \\ Department of Physics, University of Warwick, Coventry CV4 7AL, UK
}

(Received 20 November 2000)

\begin{abstract}
We consider the motion of charged particles in a static magnetic reversal with a shear component, which has application for the stability of current sheets, such as in the Earth's geotail and in solar flares. We examine how the topology of the phase space changes as a function of the shear component $b_{y}$. At zero $b_{y}$, the phase space may be characterized by regions of stochastic and regular orbits (KAM surfaces). Numerically, we find that as we vary $b_{y}$, the position of the periodic orbit at the centre of the KAM surfaces changes. We use multiple-timescale perturbation theory to predict this variation analytically. We also find that for some values of $b_{y}$, all the KAM surfaces are destroyed owing to a resonance effect between two timescales, making the phase space globally chaotic. By investigating the stability of the solutions in the vicinity of the fixed point, we are able to predict for what values of $b_{y}$ this happens and when the KAM surfaces reappear.
\end{abstract}

\section{Introduction}

Particles moving in the current sheet will, in a collisionless plasma, carry the current that supports it. Of relevance to the stability of current sheets under slow change is an understanding of single-particle dynamics in static field reversals. Simple models for such a field are magnetic reversals of the form

$$
\mathbf{b}=\left[f(z), b_{y}, b_{z}\right]
$$

where $f(z)$ is an odd function, usually taking the form either of the Harris field (Harris 1962), for which $f(z)=\tanh z$, or of the parabolic model, for which $f(z)=z$. The parameters $b_{y}$ and $b_{z}$ are non-vanishing constants. Most of the analytical work in this paper gives the general treatment (any $f$ ). However, in order to evaluate the final integrals and to make predictions, we revert for simplicity to the parabolic approximation. The two models converge inside the reversal, but diverge when $|z|>1$, with the parabolic model not allowing particles to escape. Since our results deal mainly with trajectories lying in the vicinity of the reversal, for which $|z|<1$, the region of validity of the parabolic model is sufficient. A convective electric field $\mathbf{E}$ can also be taken into account with a de Hoffman-Teller frame transformation (de Hoffman and Teller 1950). For convenience, we work in the frame of reference where $\mathbf{E}=0$. A schematic diagram of the field lines in the coordinate system used is shown in Fig. 1.

The static $b_{y}=0$ model has been studied extensively (see e.g. Sonnerup 1971; Speiser 1978; Buchner and Zelenyi 1986; Chen and Palmadesso 1986; Wang 1994 


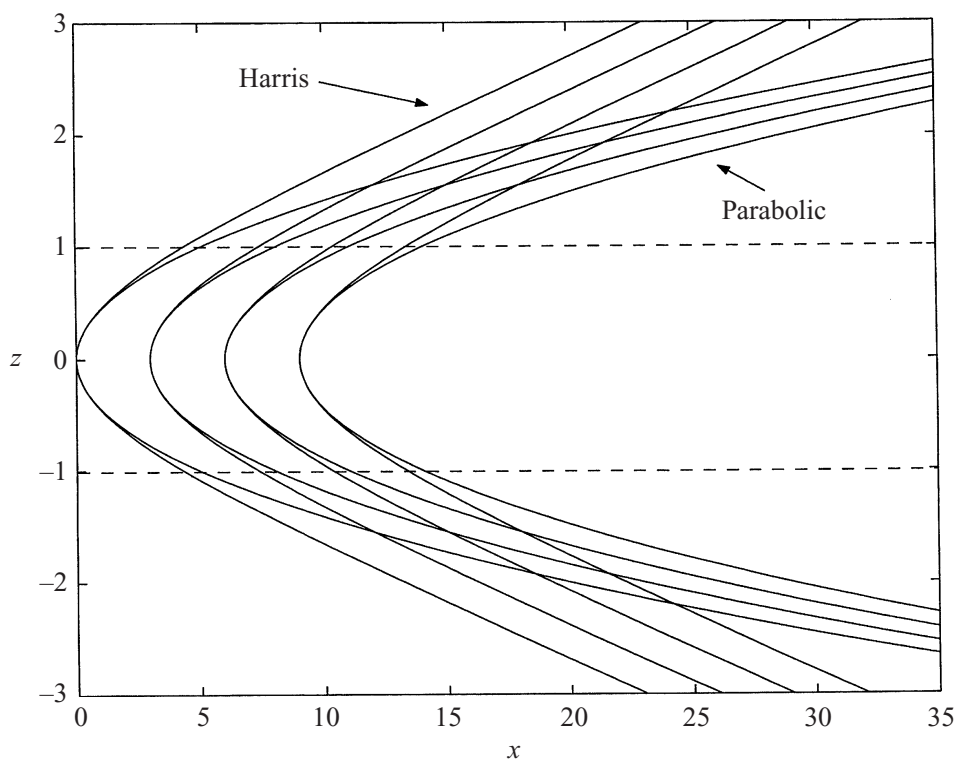

Figure 1. The coordinate system and the field lines used.

and references therein). For a comprehensive introduction to the subject, the reader is referred to the review paper by Chen (1992). The time-dependent system has, for the $b_{y}=0$ case, also been studied elsewhere (Chapman 1994).

In the static case, it was found by Chen and Palmadesso (1986) and by Buchner and Zelenyi (1986) that for non-zero $b_{z}$, the system is non-integrable, having only two global constants of the motion in involution, and that it can in general support three types of trajectory, namely regular (lying on KAM surfaces), chaotic and transient. Using Poincaré surfaces of section, it was shown that each family of trajectories covers a distinct region in phase space, the geography of which varies as a function of the field parameters and of the energy.

However, since both the geotail and solar flares have been reported to possess a finite $b_{y}$ field component (see e.g. Sergeev et al. 1993; Litvinenko 1996), it has become necessary to understand how the resulting shearing of the field lines affects the particle motion (see e.g. Karimabadi et al. 1990; Buchner and Zelenyi 1991; Zhu and Parks 1993). Recently, Chapman and Rowlands (1998) also investigated the finite- $b_{y}$ problem and obtained expressions for the bounce period and the action integral associated with the bouncing motion. They also showed that for certain $b_{y}$ intervals, all the regular trajectories are destroyed, making the phase space globally chaotic. Ynnerman et al. (2000) demonstrated that the route to global chaos in the $b_{y} \neq 0$ case is via repeated period doublings. In the same paper, it was shown that $b_{y}$ deforms the tori on which the regular trajectories lie, squashing them into a Möbius-strip-like shape.

In this paper, we investigate numerically what effects lead to the bifurcation. We also use a perturbation technique to find approximate solutions to the equations of motion and to predict how the position of the centre of the near-integrable region varies with $b_{y}$. Finally, by linearizing around the fixed point, we provide an analytical estimate for the value of $b_{y}$ at which the central orbit becomes unstable. We compare these predictions with numerical results. 


\section{The basic equations}

For a charged particle moving in the magnetic field given by (1.1), the equations of motion can be obtained from the Lorenz force law. In a suitable normalization, they are

and the energy equation is

$$
\begin{gathered}
\frac{d^{2} \phi}{d \tau^{2}}=b_{z}^{2}[F(z)-\phi]-b_{y} b_{z} \frac{d z}{d \tau}, \\
\frac{d^{2} z}{d \tau^{2}}=\frac{b_{y}}{b_{z}} \frac{d \phi}{d \tau}-f(z)[F(z)-\phi],
\end{gathered}
$$

$$
h=\frac{1}{b_{z}^{2}}\left(\frac{d \phi}{d \tau}\right)^{2}+\left(\frac{d z}{d \tau}\right)^{2}+[F(z)-\phi]^{2},,
$$

where $F(z)=\int f(z) d z, \phi=b_{z} x-p_{y}$ ( $p_{y}$ is the $y$ momentum, and the second invariant of the motion) and we have used the $y$ invariance of the field to reduce it to a two-dimensional problem. The full derivation of these equations and the normalization used can be found in Chapman and Rowlands (1998).

A convenient way to visualize the numerical solutions of these equations is the $z=0$ Poincaré surface of section, which consists of injecting into the field a large number of particles, all with the same energy $h$ and $p_{y}$, but each with a different initial condition, such that all the phase space is explored. Every time the trajectory crosses the $z=0$ plane it leaves a trace. It should be noted that while for the $b_{y}=0$ case, the $\dot{z}>0$ and the $\dot{z}<0$ surfaces of section are identical, this is no longer the case when $b_{y} \neq 0$. However, since both surfaces of section sample the same trajectories, it is sufficient to concentrate solely on one of the two. Here the $\dot{z}>0$ surface of section is used.

Examples of $z=0$ surfaces of section for $b_{y}=0$ can be found in Chen and Palmadesso (1986) and in Buchner and Zelenyi (1986). Their evolution as a function of $b_{y}$ is shown in Buchner and Zelenyi (1991) and in Chapman and Rowlands (1998), where regions of global chaos are apparent for certain values of $b_{y}$. The bifurcation sequence leading to the onset of global chaos can be found in Ynnerman et al. (2000), where it is also shown that the position of the central periodic orbit on the surface of section varies as a function of $b_{y}$.

Here we shall use as examples three values of $h$, namely $h=0.001, h=0.01$ and $h=0.1$. We shall investigate the effect of $b_{y}$ both on single trajectories and on the phase-space topology.

\section{The effects of $b_{y}$ on single trajectories}

It is instructive to examine the effect of $b_{y}$ on single trajectories. Figures 2 and 3 show the $x, y$ and $z$ components of two quasiperiodic trajectories in the vicinity of the central periodic orbit - the first for $b_{y}=0$ and the second for $b_{y}=0.005$. For both trajectories $h=0.01, p_{y}=0$ and $b_{z}=0.1$. In the corresponding power spectra (Figs 4 and 5), we see that whilst for the $b_{y}=0$ case the $x$ and $z$ oscillations are distinct (exhibiting one fast oscillation and a slower modulation), when a finite $b_{y}$ is introduced they modulate each other, with the power spectra of each component of the motion exhibiting two modulations (corresponding to the originally separate $x-z$ modulations) in addition to the fast one. The higher-frequency peaks correspond to the harmonics of the main frequencies. In the next section, we show that the behaviour of the slower modulations is crucial in determining the stability of the KAM surfaces. 

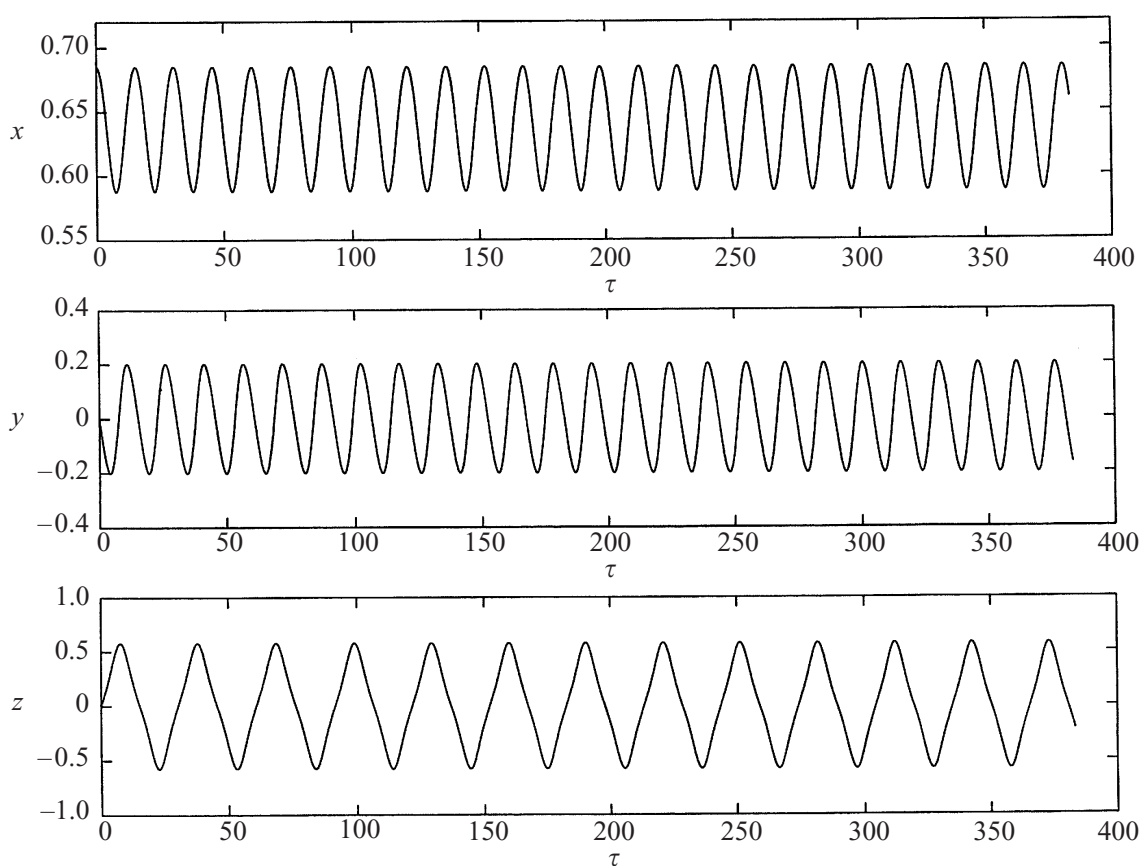

Figure 2. The $x, y$ and $z$ coordinates of a quasiperiodic orbit initiated at $x=0.6849, z=0$, $v_{x}=0$ for $h=0.01, p_{y}=0, b_{z}=0.1$ and $b_{y}=0$.
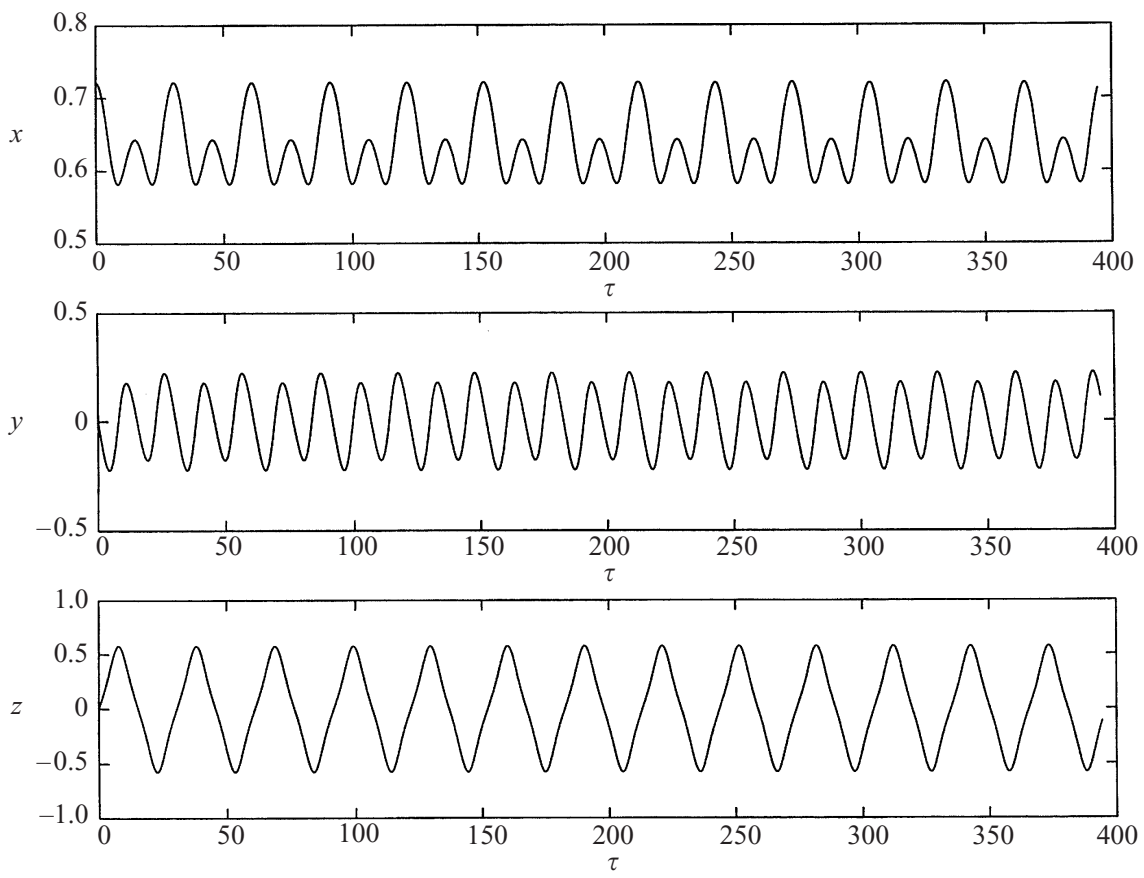

Figure 3. The $x, y$ and $z$ coordinates of a quasiperiodic orbit initiated at $x=0.72125, z=0$, $v_{x}=0$ for $h=0.01, p_{y}=0, b_{z}=0.1$ and $b_{y}=0.005$. 

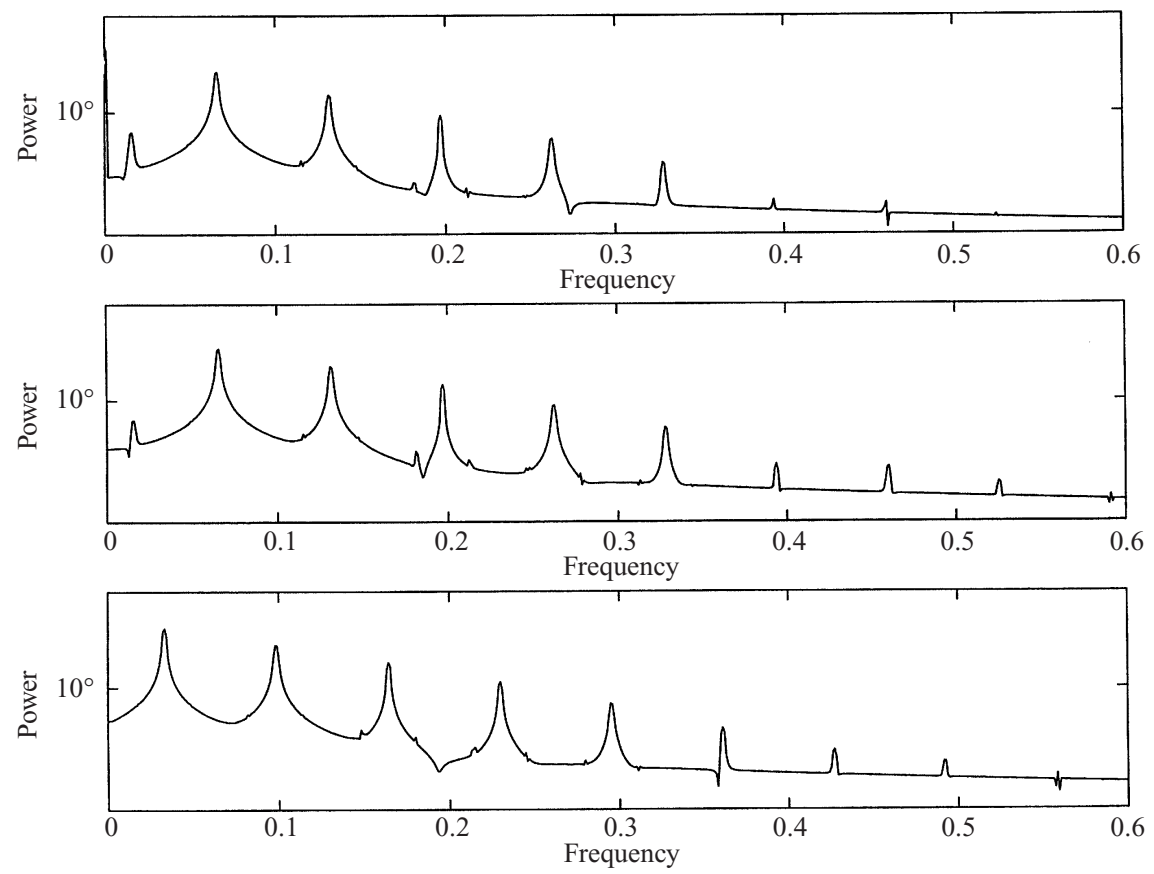

Figure 4. The power spectra of the $x, y$ and $z$ components of the motion of the orbit shown in Fig. $2\left(b_{y}=0\right)$.
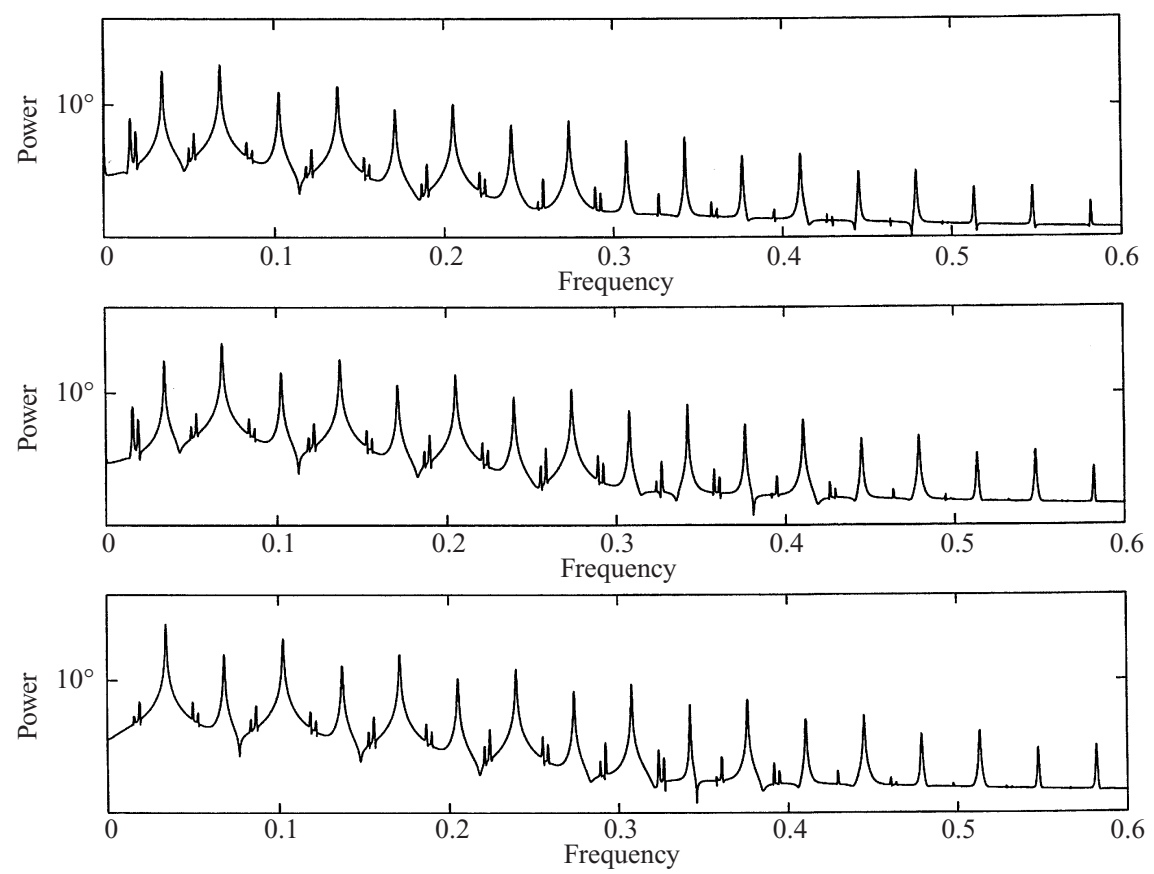

Figure 5. The power spectra of the $x, y$ and $z$ components of the motion of the orbit shown in Fig. $3\left(b_{y}=0.005\right)$. 

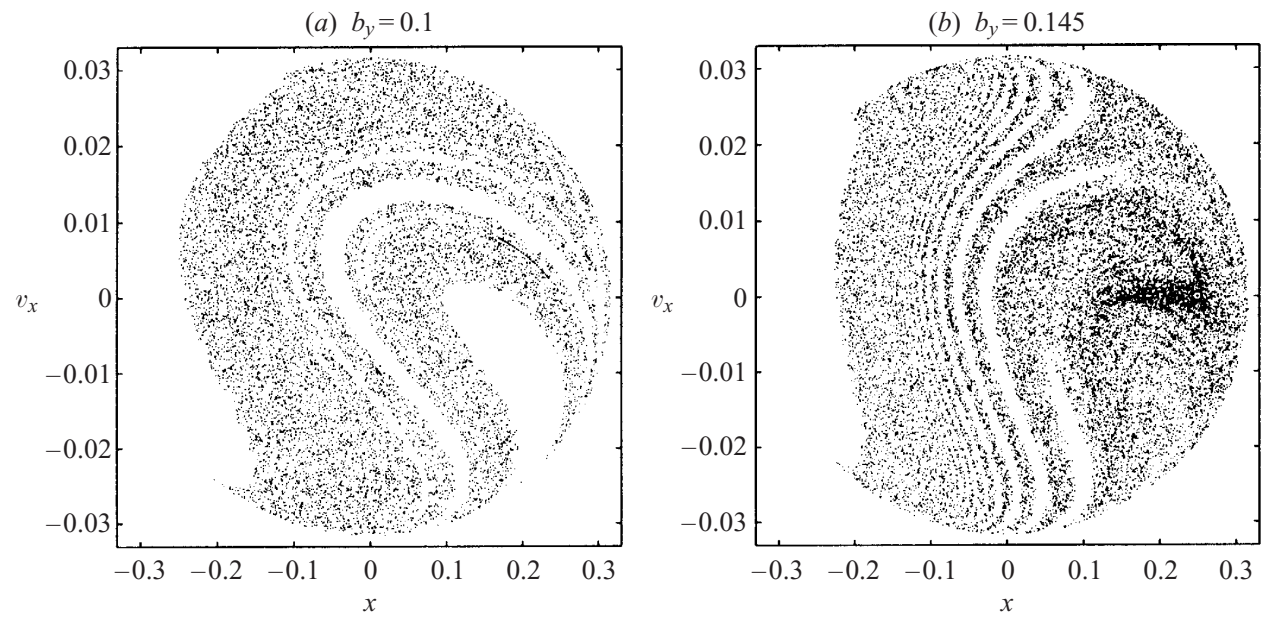

(c) $b_{y}=0.17$

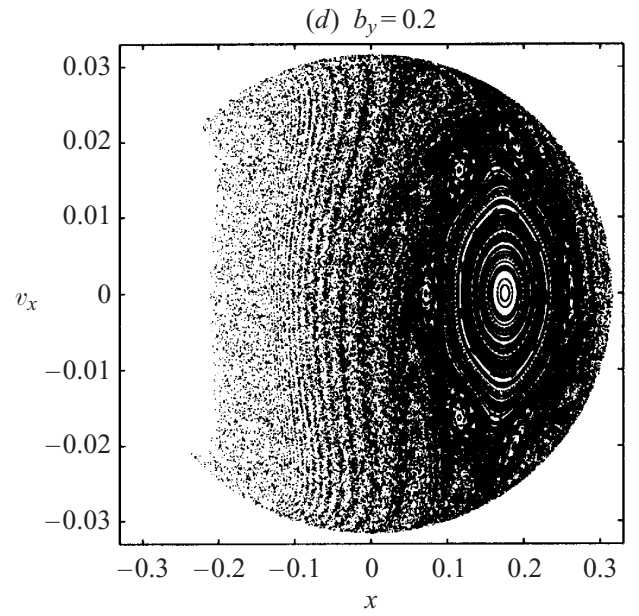

Figure 6. $z=0$ surface-of-section plots for $h=0.001$ and for increasing $b_{y}$. The other parameters are $b_{z}=0.1$ and $p_{y}=0$.

\section{The effect of $b_{y}$ on the $z=0$ surfaces of section}

For $h=0.001$, the evolution of the phase space for increasing $b_{y}$ is shown in Fig. 6 . At this energy, there is initially no quasi-integrable region (see Chen and Palmadesso 1986). However, as $b_{y}$ is increased, a quasi-integrable region appears via a pitchfork bifurcation at around $b_{y} \approx 0.145$ (see Ynnerman et al. 2000). As $b_{y}$ is further increased, the near-integrable region grows in size until it covers the whole of the surface of section (the $b_{y} \rightarrow \infty$ limit is integrable). In addition, the $x$ position of the central periodic orbit varies as a function of $b_{y}$. This variation is shown in Fig. 7, where the form of the bifurcation is shown, as determined numerically from the surfaces of section. Superimposed are the analytical results obtained in the next section.

In parallel to the surfaces of section, one can investigate how the periods in the vicinity of the central periodic orbit (when it exists) vary with $b_{y}$. This is shown in Fig. 8. The fast and slow periods were obtained by inspection of the power spectra 


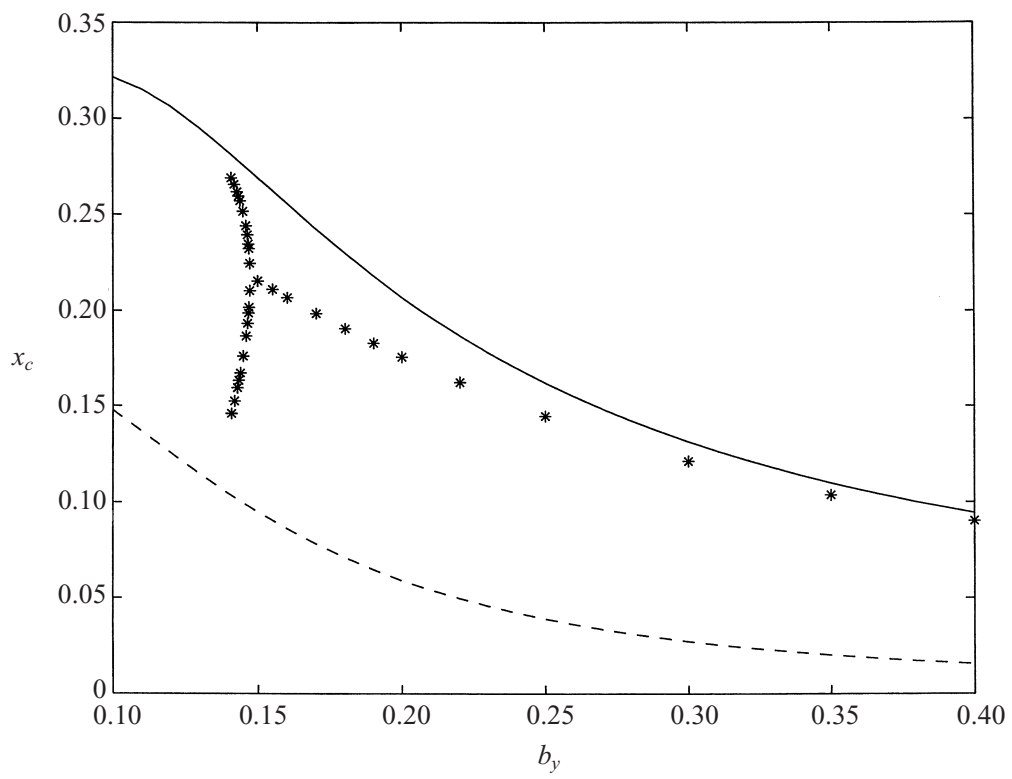

Figure 7. Comparison between the numerical $(*)$ and analytical $(-$ for $\dot{z}>0 ;---$ for $\dot{z}<0$ ) results for the $x(\phi)$ position of the central periodic orbit on $z=0$ as a function of $b_{y}$ for $h=0.001$ and $b_{z}=0.1$.

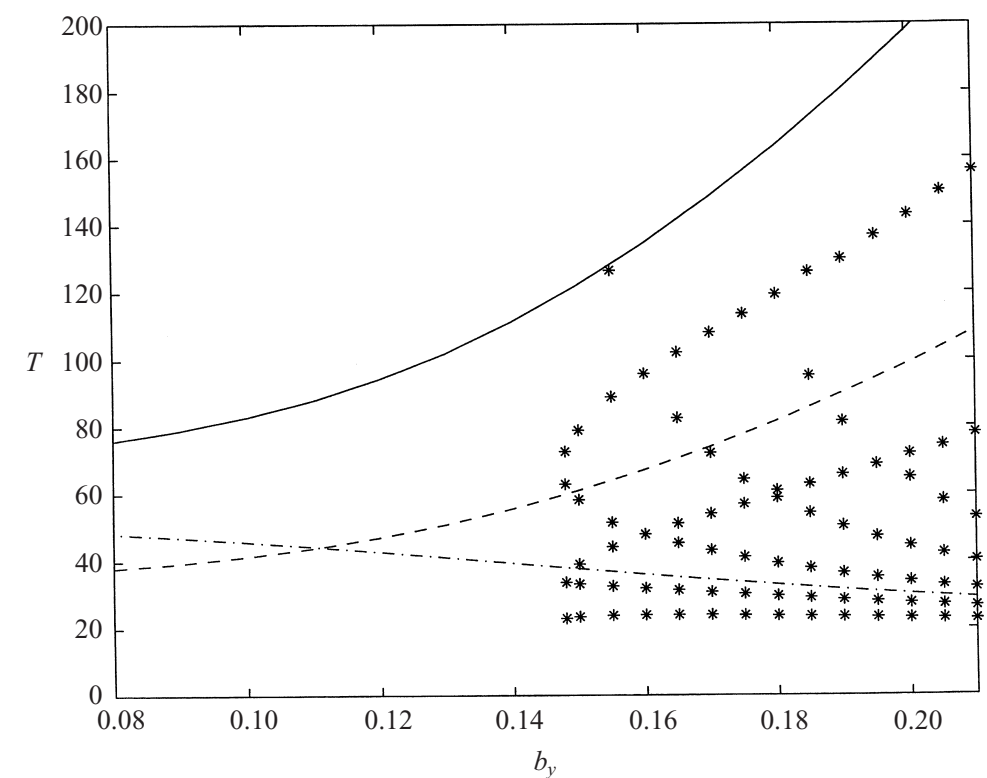

Figure 8. Comparison between numerical and analytical results for the observed periods in the vicinity of the central periodic orbit as a function of $b_{y}$ for $h=0.001$. The numerically determined periods (fast and slow) are shown with a $(*)$. For the analytical results, the continuous line (-) corresponds to $T_{s}$, the dashed line $(---)$ to $\frac{1}{2} T_{s}$ and the dashed-dotted line $(-\cdot-)$ to $\bar{T}_{f}$. 


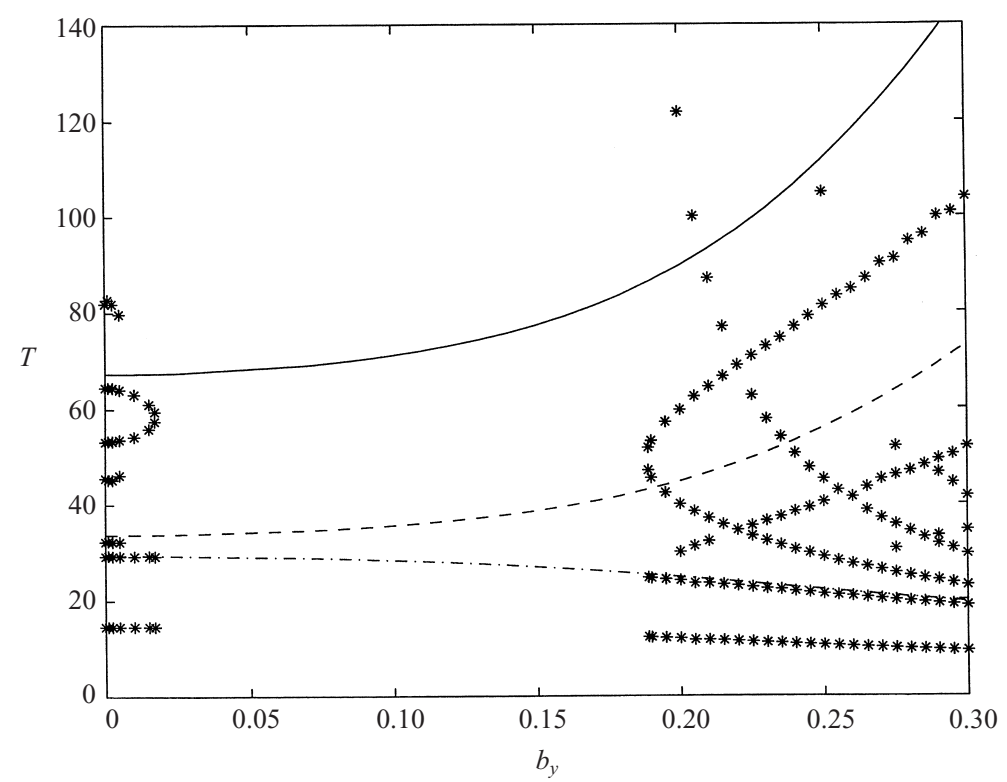

Figure 9. Comparison between the numerical $(*)$ and the analytical results for the observed periods in the vicinity of the central periodic orbit as a function of $b_{y}$ for $h=0.01$. The numerically observed periods (fast and slow) are shown with a $(*)$. For the analytical results, the continuous line $(-)$ corresponds to $T_{s}$, the dashed line $(---)$ to $\frac{1}{2} T_{s}$ and the dashed-dotted line $(-\cdot-)$ to $\bar{T}_{f}$.

of trajectories in the vicinity of the central periodic orbit for each value of $b_{y}$. At the specific value of $b_{y}$ corresponding to where bifurcation occurs, the two slow frequencies become equal. Importantly, the value towards which they converge is equal to twice the fast period. Thus it appears that the reason the fixed point becomes unstable is that the higher-order harmonics of the slow periods overlap with the fast period. This coupling leads to the destruction of all the regular trajectories. One can also observe an additional periodicity in the motion that tends to infinity near the bifurcation and is a clear signature that the periodic trajectory becomes unstable. In addition, for values of $b_{y}$ where the slow period is smaller than twice the fast period, no regular trajectories exist.

A similar argument can be used for $h=0.01$, the surfaces of section of which can be found in Chapman and Rowlands (1998) and Ynnerman et al. (2000). Here we show the evolution of the largest periods observed in Figs 4 and 5 as a function of $b_{y}$ (Fig. 9). In this case, a quasi-integrable region exists in the vicinity of $b_{y}=0$, but subsequently disappears via an inverse pitchfork bifurcation at $b_{y} \approx 0.017$, and reappears at $b_{y} \approx 0.178$. In Fig. 10 , we also show the position of the fixed point on $z=0$ as a function of $b_{y}$.

As a third example, we use $h=0.1$, for which it is found (Fig. 11) that the timescales always remain well separated and that the quasi-integrable region always exists. The position of the fixed point on $z=0$ as a function of $b_{y}$ for this energy level is shown in Fig. 12.

In the next section, we use perturbation theory to obtain approximate solutions to the equations of motion (2.1)-(2.3), in order to try to predict the observed behaviour. 


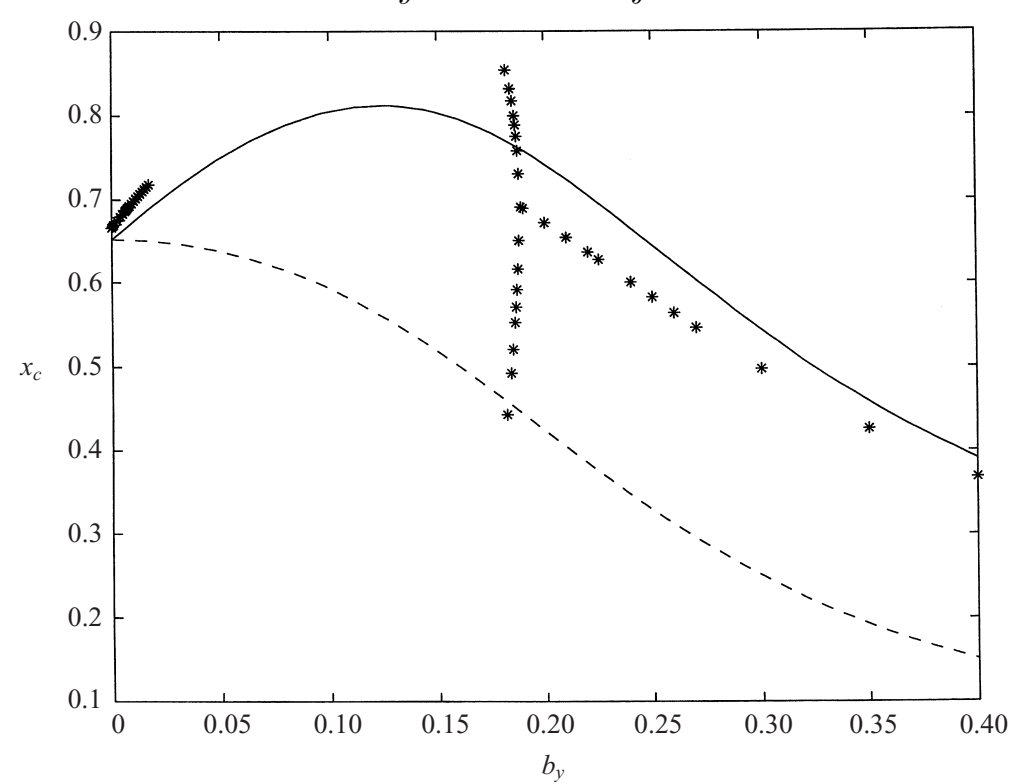

Figure 10. Comparison between the numerical $(*)$ and the analytical ( - for $\dot{z}>0$; --- for $\dot{z}<0)$ results for the $x(\phi)$ position of the central periodic orbit on $z=0$ as a function of $b_{y}$ for $h=0.01$ and $b_{z}=0.1$.

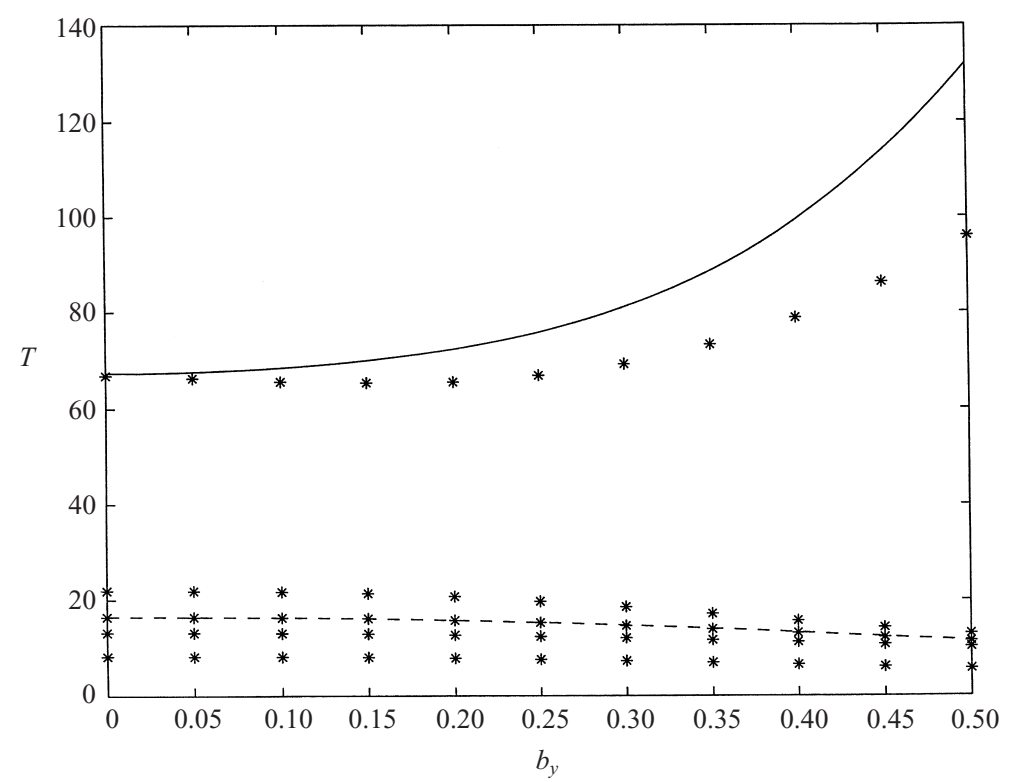

Figure 11. Comparison between the numerical $(*)$ and the analytical results for the observed periods in the vicinity of the central periodic orbit as a function of $b_{y}$ for $h=0.1$. The numerically determined periods (fast and slow) are shown with a $(*)$. For the analytical results, the continuous line (-) corresponds to $T_{s}$ and the dashed line (---) to $\bar{T}_{f}$.

\section{The perturbation procedure}

Equations (2.1)-(2.3) are nonlinear and non-integrable. To simplify them, one can use multiple-timescale perturbation theory (see e.g. Rowlands 1990). This consists 


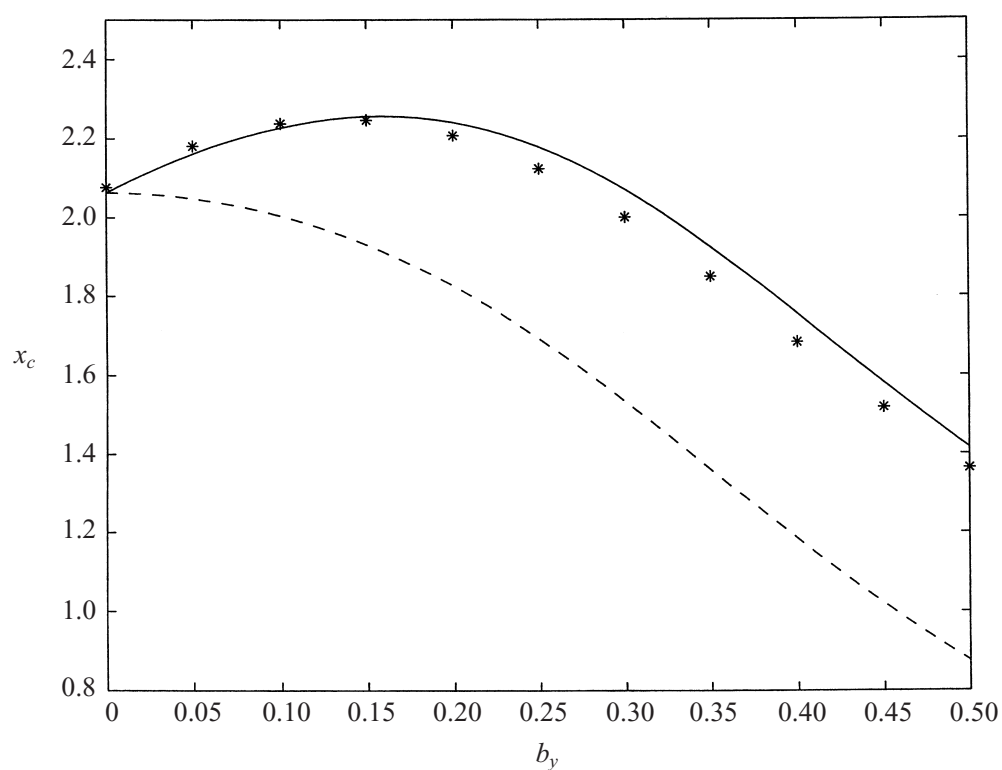

Figure 12. Comparison between numerical $(*)$ and the analytical ( - for $\dot{z}>0$; --- for $\dot{z}<0$ ) results for the $x(\phi)$ position of the central periodic orbit on $z=0$ as a function of $b_{y}$ for $h=0.1$ and $b_{z}=0.1$.

essentially of taking the oscillatory solution obtained by applying conventional perturbation theory and making the phase slowly time-varying. The formal way of doing this is to separate the timescales between the $z$ motion, which is fast, and the $x$ motion, which has a slower component. Thus one can think of a fast timescale $\left(\tau_{f}\right)$ in which a particle oscillates up and down in the $z$ axis, and a slow timescale $\left(\tau_{s}\right)$, in which the particle rotates around the KAM surface in the $(x, \dot{x})$ plane. Then, using $b_{z}$ as our expansion parameter (i.e. assuming that $b_{z} \ll 1$ ), we can write $\tau_{s}=b_{z} \tau_{f}$, and hence

$$
\frac{d}{d \tau}=\frac{d}{d \tau_{f}}+b_{z} \frac{d}{d \tau_{s}} .
$$

Thus, by expanding $z$ and $\phi$ in the form (Chapman and Rowlands 1998)

$$
\begin{aligned}
& \phi=\phi_{0}\left(\tau_{s}\right)+b_{z} \phi_{1}\left(\tau_{f}, \tau_{s}\right)+b_{z}^{2} \phi_{2}\left(\tau_{f}, \tau_{s}\right)+\ldots, \\
& z=z_{0}\left(\tau_{f}, \tau_{s}\right)+b_{z} z_{1}\left(\tau_{f}, \tau_{s}\right)+b_{z}^{2} z_{2}\left(\tau_{f}, \tau_{s}\right)+\ldots,
\end{aligned}
$$

the equations of motion yield, to lowest order,

$$
\begin{gathered}
\frac{d^{2} \phi_{0}}{d \tau_{f}^{2}}=0 \\
\frac{d^{2} z_{0}}{d \tau_{f}^{2}}=b_{y}\left(\frac{d \phi_{0}}{d \tau_{s}}+\frac{d \phi_{1}}{d \tau_{f}}\right)-f\left(z_{0}\right)\left[F\left(z_{0}\right)-\phi_{0}\right]
\end{gathered}
$$

and

$$
h_{0} \equiv h=\left(\frac{d z_{0}}{d \tau_{f}}\right)^{2}+\left(\frac{d \phi_{0}}{d \tau_{s}}+\frac{d \phi_{1}}{d \tau_{f}}\right)^{2}+\left[F\left(z_{0}\right)-\phi_{0}\right]^{2} .
$$


To order $b_{z}$,

$$
\frac{d^{2} \phi_{1}}{d \tau_{f}^{2}}=-b_{y} \frac{d z_{0}}{d \tau_{f}}
$$

And to order $b_{z}^{2}$,

$$
\frac{d^{2} \phi_{0}}{d \tau_{s}^{2}}+2 \frac{d^{2} \phi_{1}}{d \tau_{f} d \tau_{s}}+\frac{d^{2} \phi_{2}}{d \tau_{f}^{2}}=\left[F\left(z_{0}\right)-\phi_{0}\right]-b_{y}\left(\frac{d z_{0}}{d \tau_{s}}+\frac{d z_{1}}{d \tau_{f}}\right) .
$$

Chapman and Rowlands (1998) solved (5.2)-(5.6), by showing that the action integral $\oint\left(d z_{0} / d \tau_{f}\right) d z_{0}$ is, to lowest order, a constant of the motion. In this paper, we are primarily interested in the bifurcation process, so we can use linear perturbation theory about the Chapman and Rowlands solution. Thus we can write

$$
\begin{aligned}
\phi_{0}\left(\tau_{s}\right) & =\bar{\phi}_{0}+\delta \phi_{0}\left(\tau_{s}\right), \\
z_{0}\left(\tau_{f}, \tau_{s}\right) & =\bar{z}_{0}\left(\tau_{f}\right)+\delta z_{0}\left(\tau_{f}, \tau_{s}\right),
\end{aligned}
$$

where $\bar{\phi}_{0}$ is now a strict constant during an oscillation, corresponding to the position of the central periodic orbit, and $\bar{z}_{0}\left(\tau_{f}\right)$ corresponds to $z_{0}$ on $\bar{\phi}_{0}$. The small element $\delta \phi_{0}\left(\tau_{s}\right)$ is the linearization about the central orbit. Expanding $\phi_{1}, \phi_{2}, \ldots$ and $z_{1}, z_{2}, \ldots$ in the same way and substituting into (5.2)-(5.6) yields for $\bar{\phi}_{0}, \bar{\phi}_{1}, \bar{\phi}_{2}, \bar{z}_{0}$ and $\bar{z}_{1}$

$$
\begin{gathered}
\frac{d^{2} \bar{z}_{0}}{d \tau_{f}^{2}}=b_{y} \frac{d \bar{\phi}_{1}}{d \tau_{f}}-f\left(\bar{z}_{0}\right)\left[F\left(\bar{z}_{0}\right)-\bar{\phi}_{0}\right], \\
\bar{h}_{0} \equiv h_{0}=\left(\frac{d \bar{z}_{0}}{d \tau_{f}}\right)^{2}+\left(\frac{d \bar{\phi}_{1}}{d \tau_{f}}\right)^{2}+\left[F\left(\bar{z}_{0}\right)-\bar{\phi}_{0}\right]^{2}, \\
\frac{d^{2} \bar{\phi}_{1}}{d \tau_{f}^{2}}=-b_{y} \frac{d \bar{z}_{0}}{d \tau_{f}}
\end{gathered}
$$

and

$$
\frac{d^{2} \bar{\phi}_{2}}{d \tau_{f}^{2}}=\left[F\left(\bar{z}_{0}\right)-\bar{\phi}_{0}\right]-b_{y} \frac{d \bar{z}_{1}}{d \tau_{f}} .
$$

Similarly, for $\delta \phi_{0}, \delta \phi_{1}, \delta \phi_{2}, \delta z_{0}$ and $\delta z_{1}$, we obtain the equations

$$
\begin{gathered}
\frac{d^{2} \delta z_{0}}{d \tau_{f}^{2}}=b_{y}\left(\frac{d \delta \phi_{0}}{d \tau_{s}}+\frac{d \delta \phi_{1}}{d \tau_{f}}\right)-\frac{d}{d \bar{z}_{0}}\left\{f\left(\bar{z}_{0}\right)\left[F\left(\bar{z}_{0}\right)-\bar{\phi}_{0}\right]\right\} \delta z_{0}+f\left(\bar{z}_{0}\right) \delta \phi_{0} \\
\frac{d^{2} \delta \phi_{1}}{d \tau_{f}^{2}}=-b_{y} \frac{d \delta z_{0}}{d \tau_{f}}
\end{gathered}
$$

and

$$
\frac{d^{2} \delta \phi_{0}}{d \tau_{s}^{2}}+2 \frac{d^{2} \delta \phi_{1}}{d \tau_{f} d \tau_{s}}+\frac{d^{2} \delta \phi_{2}}{d \tau_{f}^{2}}=\left[f\left(\bar{z}_{0}\right) \delta z_{0}-\delta \phi_{0}\right]-b_{y}\left(\frac{d \delta z_{0}}{d \tau_{s}}+\frac{d \delta z_{1}}{d \tau_{f}}\right) .
$$

As a first remark, note in (5.10) and (5.13) that $b_{y}$ couples the first-order component of the $\phi(\tau)$ motion with the lowest-order component of the $z$ motion. This explains the coupling of the $x$ and $z$ power spectra that were found numerically. 


\section{The position of the central periodic orbit}

We first consider the solution to lowest order that corresponds to a periodic orbit passing through the $\bar{z}_{0}=0$ plane on $\bar{\phi}_{0}$. Equation (5.10) can be integrated once to give

$$
\frac{d \bar{\phi}_{1}}{d \tau_{f}}=-b_{y} \bar{z}_{0}+\bar{K},
$$

where $\bar{K}$ is the constant of integration. Since we are looking for the periodic solutions of $\bar{z}_{0}$ (period $\bar{T}_{f}$ ), we demand that $\bar{\phi}_{1}$ is also periodic with the same fast period; that is, $\bar{\phi}_{1}\left(\tau_{f}\right)=\bar{\phi}_{1}\left(\tau_{f}+\bar{T}_{f}\right)$. By integrating (6.1) over a period, we obtain for $\bar{K}$

$$
\bar{K}=\frac{b_{y}}{\bar{T}_{f}} \int_{0}^{\bar{T}_{f}} \bar{z}_{0} d \tau_{f} .
$$

It follows that if $\bar{z}_{0}$ is symmetric over a period then $\bar{K}=0$. This symmetry can be observed in the numerical integration of the full equations (Figs 2 and 3), and is a feature only of the central periodic orbit, that is of $\bar{z}_{0}\left(\tau_{f}\right)$, and not of any near-integrable orbit $z_{0}\left(\tau_{f}, \tau_{s}\right)$. By substituting for $d \bar{\phi}_{1} / d \tau_{f}$ into (5.9), we obtain

$$
d \tau_{f}= \pm \frac{d \bar{z}_{0}}{\sqrt{\bar{h}_{0}-b_{y}^{2} \bar{z}_{0}^{2}-\left[F\left(\bar{z}_{0}\right)-\bar{\phi}_{0}\right]^{2}}},
$$

and the fast period will be twice the time the particle needs to go from one extreme to the other:

$$
\bar{T}_{f}=2 \int_{-\bar{z}_{0}}^{\bar{z}_{c}} \frac{d \bar{z}_{0}}{\sqrt{\bar{h}_{0}-b_{y}^{2} \bar{z}_{0}^{2}-\left[F\left(\bar{z}_{0}\right)-\bar{\phi}_{0}\right]^{2}}},
$$

where $\bar{z}_{c}$ is the positive root of the denominator, that is, where $d \bar{z}_{0} / d \tau_{f}=0$. The positive root of (6.3) is used, since the integration is in the positive $d \bar{z}_{0} / d \tau_{f}$ direction. To evaluate this integral, we choose the parabolic approximation, for which $f\left(\bar{z}_{0}\right)=\bar{z}_{0}$. Then

$$
\bar{z}_{c}=\sqrt{2\left(\sqrt{D}+\bar{\phi}_{0}-b_{y}^{2}\right)},
$$

where

$$
D=\left(\bar{\phi}_{0}-b_{y}^{2}\right)^{2}+\bar{h}_{0}-\bar{\phi}_{0}^{2}
$$

and the integral in (6.4) can be evaluated to yield

$$
\bar{T}_{f}=8 \bar{g} K(\bar{k}),
$$

where, as before, $K(\bar{k})$ is the complete elliptic integral of the first kind,

$$
\bar{k}=\sqrt{\frac{1}{2}+\frac{\bar{\phi}_{0}-b_{y}^{2}}{2 \sqrt{D}}}
$$

and

$$
\bar{g}=\sqrt{\frac{1}{4 \sqrt{D}}}
$$

In the same way, by integrating $(6.3)$ from $\tau_{f}$ to $\tau_{f c}$ (corresponding to $\bar{z}_{c}$ ), we obtain

$$
\tau_{f c}-\tau_{f}=2 \bar{g} F\left(\arccos \frac{\bar{z}_{0}}{z c}, \bar{k}\right),
$$


and so

$$
\bar{z}_{0}=\bar{z}_{c} \operatorname{cn}\left(\frac{\tau_{f}-\tau_{f c}}{2 \bar{g}}, \bar{k}\right),
$$

where $\tau_{f c}$ can be obtained by setting an initial condition for $z$. Choosing $\bar{z}_{0}=0$ on $\tau_{f}=0$ yields

$$
\tau_{f c}=2 \bar{g} K(\bar{k})
$$

Note that the solution of $\bar{z}_{0}$ is symmetric in time, which is consistent with taking $\bar{K}=0$.

Now, in order to obtain an expression for the position of the central periodic orbit as a function of $b_{y}$, it is noted that (5.11) can be integrated over a period, and by insisting that $\bar{\phi}_{2}$ and $\bar{z}_{1}$ are periodic with period $\bar{T}_{f}$, we obtain the following equation for $\bar{\phi}_{0}$ :

$$
\bar{\phi}_{0}=\frac{2}{\bar{T}_{f}} \int_{-\bar{z}_{c}}^{\bar{z}_{c}} \frac{F\left(\bar{z}_{0}\right) d \bar{z}_{0}}{\sqrt{\bar{h}_{0}-b_{y}^{2} \bar{z}_{0}^{2}-\left[F\left(\bar{z}_{0}\right)-\bar{\phi}_{0}\right]^{2}}} .
$$

Again, for $f\left(\bar{z}_{0}\right)=\bar{z}_{0}$, the integral can be evaluated, and we find

$$
\bar{\phi}_{0}=2 \sqrt{D}\left[\frac{E(k)}{K(k)}-\frac{1}{2}\right]+\left(\bar{\phi}_{0}-b_{y}^{2}\right) .
$$

Unfortunately, it is not possible to solve this equation analytically for $\bar{\phi}_{0}$. By solving it numerically for the same three values of $\bar{h}_{0}$ used in the numerical simulations and as a function of $b_{y}$, we obtain the dashed lines in Figs 7, 10 and 12 .

Since we have demanded that $\bar{\phi}_{0}$ be a strict constant, the difference between the position of the particle as it crosses the $z=0$ plane going up and going down is not apparent. Therefore, in order to obtain a better approximation for the position of the central orbit and to show the asymmetry between the $\dot{z}>0$ and the $\dot{z}<0$ diagrams, it is necessary to go to higher order, that is to look at $\bar{\phi}_{1}$. By integrating (6.1) over $\tau_{f}$ (for $\bar{K}=0$ ), we obtain for $\bar{\phi}_{1}$ the expression

$$
\bar{\phi}_{1}=-b_{y} \int^{\tau_{f}} \bar{z}_{0} \mathrm{~d} \tau_{f}+\bar{C},
$$

where $\bar{C}$ is the constant of integration. The integral can be evaluated for $f\left(\bar{z}_{0}\right)=\bar{z}_{0}$, to yield

$$
\bar{\phi}_{1}=-2 b_{y} \arccos \left[\sqrt{1-k^{2} \operatorname{sn}^{2}\left(\frac{\tau_{f}-\tau_{f c}}{2 \bar{g}}, \bar{k}\right)}\right]+\bar{C} .
$$

To obtain a value for the constant $\bar{C}$, we observe from the form of the expected trajectory (Fig. 13) that $\bar{\phi}_{1}\left(\tau_{f}=\frac{1}{4} \bar{T}_{f}\right)=0$, which yields $\bar{C}=0$. After some simplifications, we obtain for the value of $\bar{\phi}_{1}$ on $\bar{z}_{0}=0$

$$
\left.\bar{\phi}_{1}\right|_{\bar{z}_{0}=0}=-b_{y} \arccos \left(-\frac{\bar{\phi}_{0}-b_{y}^{2}}{\sqrt{D}}\right) .
$$

The continuous lines in Figs 7, 10 and 12 then correspond to $\bar{\phi}_{0}+\left.b_{z} \bar{\phi}_{1}\right|_{\bar{z}_{0}=0}$. We see that they are in relatively good agreement (to order $b_{z}^{2}$ ) with the numerical results. It should also be noted that the position of the fixed point on the equivalent $\dot{z}<0$ surfaces of section can also be obtained, being $\bar{\phi}_{0}-\left.b_{z} \phi_{1}\right|_{\bar{z}_{0}=0}$. Of course, the agree- 


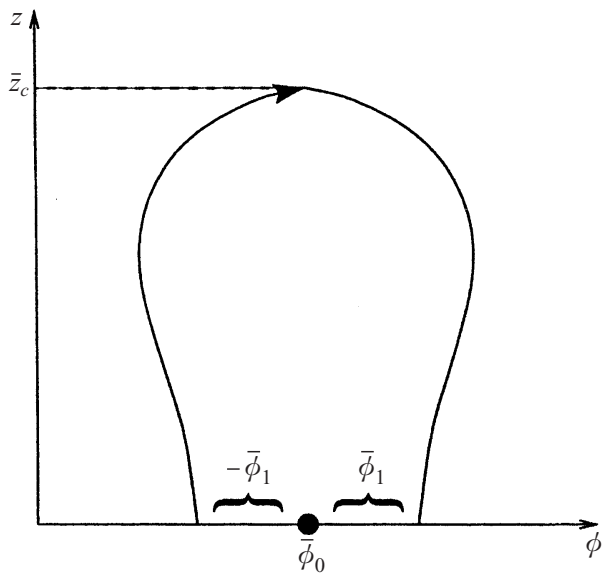

Figure 13. The path of integration used to calculate $\left.\bar{\phi}_{1}\right|_{\bar{z}_{0}=0}$.

ment between numerical and analytical results is best far from the bifurcations, since perturbation theory is no longer valid in their vicinity.

\section{The stability of the central periodic orbit}

To investigate the stability of the central periodic orbit, we must look at the slow variation $\delta \phi_{0}\left(\tau_{s}\right)$. To do this, we integrate (5.6) over a fast period $T_{f}\left(\tau_{s}\right)$, insisting that $\phi_{1}\left(\tau_{f}, \tau_{s}\right), \phi_{2}\left(\tau_{f}, \tau_{s}\right)$ and $z_{1}\left(\tau_{f}, \tau_{s}\right)$ be periodic in the fast period; that is, $\phi_{1}\left(\tau_{f}, \tau_{s}\right)=\phi_{1}\left(\tau_{f}+T_{f}\left(\tau_{s}\right), \tau_{s}\right)$, etc. We obtain the equation

$$
\frac{d^{2} \phi_{0}}{d \tau_{s}^{2}}=\frac{1}{T_{f}\left(\tau_{s}\right)} \int_{0}^{T_{f}\left(\tau_{s}\right)} d \tau_{f}\left[F\left(z_{0}\right)-\phi_{0}-b_{y} \frac{d z_{0}}{d \tau_{s}}\right],
$$

and by linearizing as before, using (5.7), we obtain for $\bar{\phi}_{0}$ equation (5.11), and for $\delta \phi_{0}$ the expression

$$
\frac{d^{2} \delta \phi_{0}}{d \tau_{s}^{2}}+\delta \phi_{0}=\frac{1}{\bar{T}_{f}} \int_{0}^{\bar{T}_{f}} d \tau_{f}\left[f\left(\bar{z}_{0}\right) \delta z_{0}-b_{y} \frac{d \delta z_{0}}{d \tau_{s}}\right]-\bar{\phi}_{0} \frac{\delta T_{f}}{\bar{T}_{f}},
$$

where

$$
T_{f}\left(\tau_{s}\right)=\bar{T}_{f}+\delta T_{f}\left(\tau_{s}\right)
$$

In order to evaluate the integral on the right-hand side, an expression for $\delta z_{0}$ is required. To obtain it, we begin by integrating (5.13) once. This gives

$$
\frac{d \delta \phi_{1}}{d \tau_{f}}=-b_{y} \delta z_{0}+\delta K\left(\tau_{s}\right)
$$

where $\delta K\left(\tau_{s}\right)$ is an integration constant given by

$$
\delta K\left(\tau_{s}\right)=b_{y}\left\langle\delta z_{0}\right\rangle
$$

where from now on the notation used is

$$
\langle\ldots\rangle=\frac{1}{\bar{T}_{f}} \int_{0}^{\bar{T}_{f}} \ldots d \tau_{f}
$$


In addition, substituting (7.4) into (5.12) yields

$$
L \delta z_{0}=b_{y}\left(\frac{d \delta \phi_{0}}{d \tau_{s}}+\delta K\right)+f\left(\bar{z}_{0}\right) \delta \phi_{0}
$$

where the operator $L$ is defined as

$$
L=\frac{d^{2}}{d \tau_{f}^{2}}+b_{y}^{2}+\frac{d}{d \bar{z}_{0}}\left\{f\left(\bar{z}_{0}\right)\left[F\left(\bar{z}_{0}\right)-\bar{\phi}_{0}\right]\right\} .
$$

Thus we can write

$$
\delta z_{0}=b_{y} A\left(\tau_{f}\right)\left(\frac{d \delta \phi_{0}}{d \tau_{s}}+\delta K\right)+B\left(\tau_{f}\right) \delta \phi_{0}
$$

where $A$ and $B$ are fast-varying functions, independent of the slow timescale. To obtain them, we must solve the following second-order differential equations:

$$
\begin{gathered}
L A=1, \\
L B=f\left(\bar{z}_{0}\right) .
\end{gathered}
$$

Integrating (7.8) over a fast period yields

$$
\left\langle\delta z_{0}\right\rangle=b_{y}\langle A\rangle\left(\frac{d \delta \phi_{0}}{d \tau_{s}}+\delta K\right)+\langle B\rangle \delta \phi_{0},
$$

and by combining this with (7.5) and rearranging for $\delta K\left(\tau_{s}\right)$, we obtain

$$
\delta K\left(\tau_{s}\right)=\left(\frac{b_{y}^{2}\langle A\rangle}{1-b_{y}^{2}\langle A\rangle}\right) \frac{d \delta \phi_{0}}{d \tau_{s}}+\left(\frac{b_{y}\langle B\rangle}{1-b_{y}^{2}\langle A\rangle}\right) \delta \phi_{0},
$$

Finally, by substituting for $\delta K\left(\tau_{s}\right)$ into (7.8), we obtain for $\delta z_{0}$ the expression

$$
\delta z_{0}=b_{y}\left(A+\frac{b_{y}^{2} A\langle A\rangle}{1-b_{y}^{2}\langle A\rangle}\right) \frac{d \delta \phi_{0}}{d \tau_{s}}+\left(B+\frac{b_{y}^{2} A\langle B\rangle}{1-b_{y}^{2}\langle A\rangle}\right) \delta \phi_{0} .
$$

By substituting for $\delta z_{0}$ into (7.2) and using (7.9) and (7.10), we can write (7.2) in the form

$$
\frac{d^{2} \delta \phi_{0}}{d \tau_{s}^{2}}\left(\frac{1}{1-b_{y}^{2}\langle A\rangle}\right)+\delta \phi_{0}\left(1-\left\langle f\left(\bar{z}_{0}\right) B\right\rangle-\frac{b_{y}^{2}\langle B\rangle^{2}}{1-b_{y}^{2}\langle A\rangle}\right)=-\bar{\phi}_{0} \frac{\delta T_{f}}{\bar{T}_{f}} .
$$

The details of how this form is obtained are given in Appendix A. To proceed, an expression for $\delta T_{f}\left(\tau_{s}\right)$ is required. A method to obtain it is given in Appendix B. We find

$$
\delta T_{f}\left(\tau_{s}\right)=\frac{2 K(\bar{k})}{D^{5 / 4}}\left[b_{y}^{2}+\frac{\bar{\phi}_{0}\left(\bar{h}_{0}-b_{y}^{2} \bar{\phi}_{0}\right)}{\bar{h}_{0}-\bar{\phi}_{0}^{2}}\right] \delta \phi_{0} .
$$

In addition, the integrals $\langle A\rangle,\langle B\rangle$ and $\left\langle f\left(\bar{z}_{0}\right) B\right\rangle$ need to be evaluated. This is done in Appendix C, where we find for the parabolic approximation

$$
\begin{gathered}
\langle A\rangle=\frac{b_{y}^{2}}{D}, \\
\langle B\rangle=0
\end{gathered}
$$

and

$$
\left\langle f\left(\bar{z}_{0}\right) B\right\rangle=\frac{\bar{h}_{0}}{2\left(\bar{h}_{0}-\bar{\phi}_{0} b_{y}^{2}\right)} .
$$


By substituting these into (7.14), we obtain, after some simplifications,

$$
\frac{d^{2} \delta \phi_{0}}{d \tau_{s}^{2}}+\omega_{s}^{2} \delta \phi_{0}=0
$$

Thus, to lowest order, the variation of $\delta \phi_{0}$ is simple-harmonic, with frequency

$$
\omega_{s}^{2}=\frac{\bar{h}_{0}-2 \bar{\phi}_{0} b_{y}^{2}}{2 D}\left[\frac{\bar{h}_{0}-2 \bar{\phi}_{0} b_{y}^{2}}{\bar{h}_{0}-\bar{\phi}_{0} b_{y}^{2}}+\frac{\bar{\phi}_{0}\left(\bar{h}_{0} \bar{\phi}_{0}-2 \bar{\phi}_{0}^{2} b_{y}^{2}+\bar{h}_{0} b_{y}^{2}\right)}{\left(\bar{h}_{0}-\bar{\phi}_{0}^{2}\right) D}\right] .
$$

The slow period is then given by

$$
T_{s}=\frac{2 \pi}{b_{z} \omega_{s}} .
$$

The analytically obtained periods and their harmonics are shown in Figs 8-11, superimposed on the numerical data. In all three cases, the analytical expression for $T_{s}$ is qualitatively accurate, but it predicts higher values than expected from the numerical results. Also, the analytical predictions are more accurate for higher energies.

There is a good explanation for these discrepancies. One should keep in mind the fact that we had to linearize, a procedure that, as we saw in the $b_{y}=0$ case, is not very accurate in the vicinity of a bifurcation. Buchner and Zelenyi (1986) showed for $b_{y}=0$ that the value obtained for the slow period corresponds to the value towards which the actual solution converges for large $h$. The fact that the accuracy of our results increases with increasing $h$ suggests that this is still true when a $b_{y}$ component is introduced. However, the growing discrepancy between analytical and numerical values of $T_{s}$ for increasing $b_{y}$ suggests that the convergence rate is slower for large values of $b_{y}$. In contrast, the prediction for $\bar{T}_{f}$ is reasonable, with the accuracy again increasing for increasing $h$.

Numerically, we found that the destruction of the near-integrable region via a bifurcation occurs when the two slow periods (one of which we have obtained here, the other one being of higher order, corresponding to the slow periods of $z_{1}$ and $\phi_{2}$ ) become equal to twice the fast period, leading to a resonance between the fast period and a higher-order harmonic of the slow period. In Fig. 8 , we see that $\bar{T}_{f} \approx \frac{1}{2} T_{s}$ at $b_{y} \approx 0.1108$, and this is therefore where we should expect the bifurcation to occur. The actual value where it occurs is $b_{y} \approx 0.145$. The difference is mainly due to our large error in the calculation of $T_{s}$.

For the $h=0.01$ case, we see that our approximation does not allow us to find the bifurcation, since, contrary to what is observed numerically, we find $\frac{1}{2} T_{s}$ to be always greater than $T_{f}$. Again, this is due to our high estimate of $T_{s}$. The sudden decrease in $T_{s}$ observed numerically at $b_{y} \approx 0.01$ is probably due to higher-order effects that are not included in the lowest-order approximation.

Finally, in Fig. 11, we observe that for $h=0.1$, the prediction for $T_{s}$ is more accurate than in the previous two cases and that all the timescales remain well separated.

\section{Conclusions}

The effects of a $b_{y}$ component on the motion of charged particles in a magnetic reversal have been studied both numerically and analytically, and the effects leading to the bifurcation observed by Ynnerman et al. (2000) investigated. We have 
found that bifurcations occur when the fast and the slow timescales resonate. We have also shown that as $b_{y}$ increases, the $x$ position of the central periodic orbit in the Poincaré surface of section varies. This variation has been obtained here analytically by using a multiple-timescale perturbation technique around the $b_{z}=0$ solution. By linearizing around this fixed point, we have also provided analytical estimates (in some cases) for the value of $b_{y}$ at which quasiperiodic orbits reappear via a bifurcation. These estimates correctly quantify the resonance effect leading to the onset of global chaos.

\section{Appendix A. Derivation of (7.14)}

By inserting (7.13) into (7.2) and using (7.9) and (7.10), we obtain the following coefficients for each term on the right-hand side of $(7.2)$ :

(a) for $d^{2} \delta \phi_{0} / d \tau_{s}^{2}$, the coefficient is

$$
\left\langle-b_{y}\left(b_{y} A+\frac{b_{y}^{3} A\langle A\rangle}{1-b_{y}^{2}\langle A\rangle}\right)\right\rangle
$$

which simplifies to

$$
-\frac{b_{y}^{2}\langle A\rangle}{1-b_{y}^{2}\langle A\rangle}
$$

(b) for $d \delta \phi_{0} / d \tau_{s}$, it is

$$
\left\langle L B\left(b_{y} A+\frac{b_{y}^{3} A\langle A\rangle}{1-b_{y}^{2}\langle A\rangle}\right)-b_{y}\left(B+\frac{b_{y}^{2} A\langle B\rangle}{1-b_{y}^{2}\langle A\rangle}\right)\right\rangle,
$$

which is equal to zero, since $\langle L B A\rangle=\langle L A B\rangle=\langle B\rangle$;

(c) finally, the coefficient for $\delta \phi_{0}$ is

$$
\left\langle L B\left(B+\frac{b_{y}^{2} A\langle B\rangle}{1-b_{y}^{2}\langle A\rangle}\right)\right\rangle
$$

which simplifies to

$$
\left\langle f\left(\bar{z}_{0}\right) B\right\rangle+\frac{b_{y}^{2}\langle B\rangle^{2}}{1-b_{y}^{2}\langle A\rangle} .
$$

By putting all these terms together in (7.2), we obtain

$$
\frac{d^{2} \delta \phi_{0}}{d \tau_{s}^{2}}+\delta \phi_{0}=\left(-\frac{b_{y}^{2}\langle A\rangle}{1-b_{y}^{2}\langle A\rangle}\right) \frac{d^{2} \delta \phi_{0}}{d \tau_{s}^{2}}+\left(\left\langle f\left(\bar{z}_{0}\right) B\right\rangle+\frac{b_{y}^{2}\langle B\rangle^{2}}{1-b_{y}^{2}\langle A\rangle}\right) \delta \phi_{0}-\bar{\phi}_{0} \frac{\delta T_{f}}{\bar{T}_{f}}
$$

which, after some simplifications, yields (7.14).

\section{Appendix B. Obtaining an expression for $\delta T_{f}$}

By integrating (5.5) once and substituting the expression obtained for $d \phi_{1} / d \tau_{f}$ into (5.4), we obtain, after some manipulations,

$$
d \tau_{f}=\frac{d z_{0}}{\sqrt{h_{0}-\left[b_{y} z_{0}-d \phi_{0} / d \tau_{s}-K\left(\tau_{s}\right)\right]^{2}-\left[F\left(z_{0}\right)-\phi_{0}\right]^{2}}}
$$


where $K\left(\tau_{s}\right)$ is the integration constant obtained from the integration of (5.5). From our linearization, we know that $K\left(\tau_{s}\right) \approx \delta K\left(\tau_{s}\right)$ (since $\bar{K}=0$ ) and that $d \phi_{0} / d \tau_{s} \approx d \delta \phi_{0} / d \tau_{s}$. By substituting for these, we write

$$
d \tau_{f}=\frac{d z_{0}}{\sqrt{h_{0}-\left(b_{y} z_{0}-\epsilon\right)^{2}-\left[F\left(z_{0}\right)-\phi_{0}\right]^{2}}}
$$

where the notation

$$
\epsilon=\frac{d \delta \phi_{0}}{d \tau_{s}}+\delta K\left(\tau_{s}\right)=\frac{1}{1-b_{y}^{2}\langle A\rangle} \frac{d \delta \phi_{0}}{d \tau_{s}}
$$

has been used. We shall assume that $\epsilon$ is small and can be used as an expansion parameter. For the parabolic approximation, (B 2) can be further simplified by writing it in the form

$$
d \tau_{f}=2 \frac{d z_{0}}{\sqrt{\left(a^{2}-z_{0}^{2}\right)\left(b^{2}+z_{0}^{2}\right)+8 \epsilon b_{y} z_{0}}},
$$

where

$$
a^{2}=2\left[\sqrt{\left(\phi_{0}-b_{y}^{2}\right)^{2}+h_{0}-\phi_{0}^{2}-\epsilon^{2}}+\phi_{0}-b_{y}^{2}\right]
$$

and

$$
b^{2}=2\left[\sqrt{\left(\phi_{0}-b_{y}^{2}\right)^{2}+h_{0}-\phi_{0}^{2}-\epsilon^{2}}-\phi_{0}+b_{y}^{2}\right] .
$$

By expanding the denominator of (B 3) for small $\epsilon$, we obtain

$$
d \tau_{f}=2 \frac{d z_{0}}{\sqrt{\left(a-z_{0}-\epsilon \gamma\right)\left(a+z_{0}+\epsilon \gamma\right)\left(z_{0}^{2}-2 \epsilon \gamma z_{0}+b^{2}\right)}}+O\left(\epsilon^{2}\right),
$$

where

$$
\gamma=-\frac{4 b_{y}}{a^{2}+b^{2}}
$$

The two roots of the denominator, corresponding to the turning points of $z_{0}$, are $-a-\epsilon \gamma$ and $a-\epsilon \gamma$. Note that in this more general case, $z_{0}$ is no longer symmetric. The fast period $T_{f}\left(\tau_{s}\right)$ is obtained by integrating twice from one turning point to the other:

$$
T_{f}\left(\tau_{s}\right)=4 \int_{-a-\epsilon \gamma}^{a-\epsilon \gamma} \frac{d z_{0}}{\sqrt{\left(a-z_{0}-\epsilon \gamma\right)\left(a+z_{0}+\epsilon \gamma\right)\left(z_{0}^{2}-2 \epsilon \gamma z_{0}+b^{2}\right)}}+O\left(\epsilon^{2}\right) .
$$

By writing $y=z_{0}+\epsilon \gamma$ and expanding the non-singular term in the denominator of (B 8), we obtain

$$
T_{f}\left(\tau_{s}\right)=4 \int_{-a}^{a} \frac{d y}{\sqrt{\left(a^{2}-y^{2}\right)\left(b^{2}+y^{2}\right)}}\left[1+\frac{2 \gamma y}{b^{2}+y^{2}} \epsilon+O\left(\epsilon^{2}\right)\right] .
$$

This integral can be evaluated to give

$$
T_{f}\left(\tau_{s}\right)=8 g K(k)+O\left(\epsilon^{2}\right),
$$

where

and

$$
k\left(\tau_{s}\right)=\frac{a}{\sqrt{a^{2}+b^{2}}}
$$

$$
g\left(\tau_{s}\right)=\frac{1}{\sqrt{a^{2}+b^{2}}} .
$$


By linearizing $z_{0}$ and $\phi_{0}$ as before and writing $k\left(\tau_{s}\right)=\bar{k}+\delta k\left(\tau_{s}\right)$ and $g\left(\tau_{s}\right)=$ $\bar{g}+\delta g\left(\tau_{s}\right)$, we obtain for $\delta k\left(\tau_{s}\right)$ and $\delta g\left(\tau_{s}\right)$

$$
\delta k\left(\tau_{s}\right)=\frac{\bar{h}_{0}-b_{y}^{2} \bar{\phi}_{0}}{4 \bar{k} D^{3 / 2}} \delta \phi_{0}
$$

and

$$
\delta g\left(\tau_{s}\right)=\frac{b_{y}^{2}}{4 D^{5 / 4}} \delta \phi_{0}
$$

and $\delta T_{f}\left(\tau_{s}\right)$ is given by

$$
\delta T_{f}\left(\tau_{s}\right)=8 K(\bar{k}) \delta g+8 \bar{g} \frac{d K(\bar{k})}{d \bar{k}} \delta k,
$$

which, after some simplifications (and using (6.14)), becomes

$$
\delta T_{f}\left(\tau_{s}\right)=\frac{2 K(\bar{k})}{D^{5 / 4}}\left[b_{y}^{2}+\frac{\bar{\phi}_{0}\left(\bar{h}_{0}-b_{y}^{2} \bar{\phi}_{0}\right)}{\bar{h}_{0}-\bar{\phi}_{0}^{2}}\right] \delta \phi_{0} .
$$

Appendix C. Evaluating the integrals $\langle A\rangle,\langle B\rangle$ and $\left\langle f\left(\bar{z}_{0}\right) B\right\rangle$

By differentiating (5.8) over the fast timescale $\tau_{f}$, we observe that

$$
L \frac{d \bar{z}_{0}}{d \tau_{f}}=0 .
$$

By writing

$$
A=\tilde{A} \frac{d \bar{z}_{0}}{d \tau_{f}}
$$

and substituting into (7.9), we obtain, after some simplifications (for $f\left(\bar{z}_{0}\right)=\bar{z}_{0}$ ), the expression

$$
\left(\frac{d \bar{z}_{0}}{d \tau_{f}}\right)^{-1} \frac{d}{d \tau_{f}}\left[\frac{d \tilde{A}}{d \tau_{f}}\left(\frac{d \bar{z}_{0}}{d \tau_{f}}\right)^{2}\right]=1,
$$

which yields, after integration

$$
\frac{d \tilde{A}}{d \tau_{f}}\left(\frac{d \bar{z}_{0}}{d \tau_{f}}\right)^{2}=\bar{z}_{0}+C_{1}
$$

or

$$
\frac{d \tilde{A}}{d \tau_{f}}=\frac{\bar{z}_{0}+C_{1}}{\left(d \bar{z}_{0} / d \tau_{f}\right)^{2}},
$$

where $C_{1}$ is the constant of integration. By integrating over a fast period and insisting that $\tilde{A}$ is periodic with period $\bar{T}_{f}$, we obtain that $C_{1}=0$, since the integral

$$
\int_{0}^{\bar{T}_{f}} \frac{\bar{z}_{0}}{\left(d \bar{z}_{0} / d \tau_{f}\right)^{2}} d \tau_{f}=0 .
$$

We know $\bar{z}_{0}$ from $(6.11)$, and therefore

$$
\frac{d \bar{z}_{0}}{d \tau_{f}}=-\frac{\bar{z}_{c}}{2 \bar{g}} \operatorname{dn}(s, \bar{k}) \operatorname{sn}(s, \bar{k}),
$$


where

$$
s=\frac{\tau_{f}}{2 \bar{g}}-K(\bar{k})
$$

By integrating (C 5) from 0 to an arbitrary time $\tau_{f}<2 \bar{g} K(\bar{k})$ (in order to avoid the singularity that exists at $\tau_{f}=2 \bar{g} K(\bar{k})$ ), we obtain

$$
\tilde{A}\left(\tau_{f}\right)-\tilde{A}(0)=\int_{0}^{\tau_{f}} \frac{\bar{z}_{0}}{\left(d \bar{z}_{0} / d \tau_{f}\right)^{2}} d \tau_{f}
$$

and by substituting into this the expressions for $\bar{z}_{0}$ and $d \bar{z}_{0} / d \tau_{f}$, we obtain, after some simplifications,

$$
\tilde{A}\left(\tau_{f}\right)-\tilde{A}(0)=\frac{8 \bar{g}^{3}}{\bar{z}_{c}} \int_{-K(\bar{k})}^{s} \frac{\operatorname{cn}(s, \bar{k})}{\operatorname{dn}^{2}(s, \bar{k}) \operatorname{sn}^{2}(s, \bar{k})} d s .
$$

The integral on the right-hand side of $(\mathrm{C} 10)$ can be evaluated, and we find for $\tilde{A}\left(\tau_{f}\right)$

$$
\tilde{A}\left(\tau_{f}\right)=\frac{8 \bar{g}^{3}}{\bar{z}_{c}} \frac{\bar{k}^{2} \operatorname{sn}^{2}(s, \bar{k})-\mathrm{dn}^{2}(s, \bar{k})}{\operatorname{sn}(s, \bar{k}) \operatorname{dn}(s, \bar{k})}+G+\tilde{A}(0)
$$

where

$$
G=\frac{8 \bar{g}^{3}}{\bar{z}_{c}} \frac{2 \bar{k}^{2}-1}{\sqrt{1-\bar{k}^{2}}}
$$

and so

$$
A\left(\tau_{f}\right)=-4 \bar{g}^{2}\left[\bar{k}^{2} \operatorname{sn}^{2}(s, \bar{k})-\operatorname{dn}^{2}(s, \bar{k})\right]+\mathrm{PF}\left(\tau_{f}\right),
$$

where PF is a periodic function with period $\bar{T}_{f}$ that vanishes when integrated over a period. Note that in this expression, the singularities at $\tau_{f}=2 \bar{g}(2 n+1) K(\bar{k})(n$ integer) have disappeared and $\tau_{f}$ can proceed to $8 \bar{g} K(\bar{k})$ (that is, to cover a whole period). Finally,

$$
\langle A\rangle=\frac{1}{\bar{T}_{f}} \int_{0}^{\bar{T}_{f}} A d \tau_{f}=-\frac{\bar{g}^{2}}{K(\bar{k})} \int_{-K(\bar{k})}^{3 K(\bar{k})}\left[\bar{k}^{2} \operatorname{sn}^{2}(s, \bar{k})-\operatorname{dn}^{2}(s, \bar{k})\right] d s
$$

and, by evaluating the integral, we obtain

$$
\langle A\rangle=8 \bar{g}^{2}\left[\frac{E(\bar{k})}{K(\bar{k})}-\frac{1}{2}\right] .
$$

By using (6.14), the above expression can be simplified to the form

$$
\langle A\rangle=\frac{b_{y}^{2}}{D} .
$$

A similar argument can be used to find $\langle B\rangle$ and $\left\langle f\left(\bar{z}_{0}\right) B\right\rangle$. By writing

$$
B=\tilde{B} \frac{d \bar{z}_{0}}{d \tau_{f}}
$$

and substituting into (7.10), we obtain in the same way as before the relationship

$$
\frac{d \tilde{B}}{d \tau_{f}}=\frac{\frac{1}{2} \bar{z}_{0}^{2}+C_{2}}{\left(d \bar{z}_{0} / d \tau_{f}\right)^{2}},
$$

where $C_{2}$ is the constant of integration. It can be found by integrating over a fast 
period and demanding periodicity for $\tilde{B}$. This gives

$$
C_{2}=-\frac{1}{2} \frac{\int_{0}^{\bar{T}_{f}} \bar{z}_{0}^{2}\left(d \bar{z}_{0} / d \tau_{f}\right)^{-2} d \tau_{f}}{\int_{0}^{\bar{T}_{f}}\left(d \bar{z}_{0} / d \tau_{f}\right)^{-2} d \tau_{f}}
$$

For $f\left(\bar{z}_{0}\right)=\bar{z}_{0}$, the integrals can be evaluated to give the following equation for $C_{2}$ :

$$
C_{2}=-\frac{\bar{z}_{c}^{2}}{2} \frac{\left(1-\bar{k}^{2}\right)[2 E(\bar{k})-K(\bar{k})]}{\left(2 \bar{k}^{2}-1\right) E(\bar{k})+\left(1-\bar{k}^{2}\right) K(\bar{k})},
$$

and, in the same way as before, we find for $\langle B\rangle$ and $\left\langle f\left(\bar{z}_{0}\right) B\right\rangle$

$$
\langle B\rangle=0
$$

and

$$
\left\langle f\left(\bar{z}_{0}\right) B\right\rangle=\frac{\left(1-\bar{k}^{2}\right)[1-2 E(\bar{k}) / K(\bar{k})]+E(\bar{k})^{2} / K(\bar{k})^{2}}{\left(2 \bar{k}^{2}-1\right) E(\bar{k}) / K(\bar{k})+\left(1-\bar{k}^{2}\right)} ;
$$

again, by using (6.14), this can be simplified to yield

$$
\left\langle f\left(\bar{z}_{0}\right) B\right\rangle=\frac{\bar{h}_{0}}{2\left(\bar{h}_{0}-\bar{\phi}_{0} b_{y}^{2}\right)} .
$$

\section{References}

Buchner, J. and Zelenyi, L. M. 1986 Deterministic chaos in the dynamics of charged particles near a magnetic field reversal. Phys. Lett. 118A, 395.

Buchner, J. and Zelenyi, L. M. 1991 Regular and chaotic particle motion in sheared magnetic field reversals. Adv. Space Res. 11, 9177.

Chapman, S. C. 1994 Properties of single particle dynamics in a parabolic magnetic reversal with general time dependence. J. Geophys. Res. 99, 5977.

Chapman, S. C. and Rowlands, G. 1998 Are particles detrapped by constant $B_{y}$ in static magnetic reversals? J. Geophys. Res. 103, 4597.

Chen, J. 1992 Nonlinear dynamics of charged particles in the magnetotail. J. Geophys. Res. 97, 15011.

Chen, J. and Palmadesso, P. J. 1986 Chaos and nonlinear dynamics of single-particle orbits in a magnetotaillike magnetic field. J. Geophys. Res. 91, 1499.

de Hoffman, F. and Teller, E. 1950 Magnetohydrodynamic shock. Phys. Rev. 80, 692.

Harris, E. G. 1962 On a plasma sheath separating regions of oppositely directed magnetic field. Nuovo Cim. 23, 115.

Karimabadi, H., Pritchett, P. L. and Coroniti, F. V. 1990 Particle orbits on two-dimensional equilibrium models for the magnetotail. J. Geophys. Res. 95, 17153.

Litvinenko, Y. E. 1996 Particle acceleration in reconnecting current sheets with a nonzero magnetic field. Astrophys. J. 462, 997.

Rowlands, G. 1990 Non-Linear Phenomena in Science and Engineering. Ellis Horwood, Chichester.

Sergeev, V. A., Mitchell, D. G., Russell, C. T. and Williams, D. J. 1993 Structure of the tail plasma/current sheet at $\sim 11 r_{e}$ and its changes in the course of a substorm. J. Geophys. Res. 98, 17345 .

Sonnerup, B. U. Ö. 1971 Adiabatic particle orbits in a magnetic null sheet. J. Geophys. Res. $\mathbf{7 6}, 2811$

Speiser, T. W. 1978 Particle trajectories in model current sheets, 1. Analytical solutions. J. Geophys. Res. $21,627$.

Wang, Z. D. 1994 Single particle dynamics of the parabolic field model. J. Geophys. Res. 99, 5949 . 
Ynnerman, A., Chapman, S. C., Tsalas, M. and Rowlands, G. 2000 Identification of symmetry breaking and a bifurcation sequence to chaos in single particle dynamics in magnetic reversals. Physica D139, 217.

Zhu, Z. and Parks, G. 1993 Particle orbits in model current sheets with a nonzero $B_{y}$ component. J. Geophys. Res. 98, 7603. 